\title{
Automatic beams detection used for LiFi car-2-car communication
}

\author{
Cosmin Stoica Spahiu, \\ Computer Science and Information \\ Technology, University of Craiova, \\ Romania \\ Email: cosmin.spahiu@edu.ucv.ro
}

\author{
Abagiu Marian \\ Computer Science and Information \\ Technology, University of Craiova, \\ Romania \\ Email: marian.abagiu@gmail.com
}

\author{
Liana Stanescu \\ Computer Science and Information \\ Technology, University of Craiova, \\ Romania \\ Email: liana.stanescu@edu.ucv.ro.
}

\begin{abstract}
The car-2-car communication feature is a must for the next generation of cars. Such a functionality will increase the road safety by enhancing the drivers awareness about road conditions and eventual obstacles. It can also be used to inform the cars coming from behind about risky situations in order to trigger early avoidance actions (e.g. breaking). The paper proposes an alternate solution to the WIFI technology which is the most studied for this purpose. The proposed solution is based on Light Fidelity (LiFi) and analyse the feasibility of such a system, from image processing point of view during automatic beams detection. The solution consists of two parts: the information sender, which is the car's LED beams, and the information receiver which is an onboard camera running a special software. This solution has the advantage of using already available car's hardware with small adaption, and only integrate the new software.
\end{abstract}

\section{INTRODUCTION}

$\mathrm{T}$ HE car-2-car communication is a functionality highly researched in the last years due to the multiple benefits that might bring, and several solutions have been proposed in this direction.

The direct communication between cars will increase road safety and reduce the traffic incidents. Based on this functionality, each car will receive enough information in advance to adapt the driving based on the surroundings, in a matter of milliseconds. The drivers can be informed about hazard situations, even before they are visible on the road.

This functionality is currently in the research phase and several solutions have been proposed. However, none of them was generally adopted by OEM producers in order to be effective on large scale. To reach this goal it is needed a standard agreed by all cars manufacturers.

The "car-2-car.org" consortium was founded, aiming to define such a solution generally applicable, where all main car manufacturers participate as active members [1]. The CAR 2 CAR Communication Consortium (C2C-CC) aims at assisting towards accident free traffic (vision zero) at the earliest possible date. The consortium does not offer on-the shelves solutions but only a standard and guidance in the implementation of a common solution that is robust and reliable enough to be used in real life traffic.

There were proposed several solutions in this direction. Among them, the WI-FI communication is widely spread, as it has been proved to be a mature enough technology and very reliable.

One of the first implementations in this direction is Volkswagen's Local Hazard Warning (LHW) [2] which is a system that uses short-range communication between cars. For example, when a car is equipped with the LHW feature it might issue a warning to other vehicles if it had broken down in the middle the highway or had been involved in a collision.

The technology used in this implementation is based on an automotive optimized variant of WLAN technology known as "ETSI ITS-G5". A car who is equipped with this technology will always be informed on such situations in advance, and it is not needed to rely anymore on GSM internet network [2]

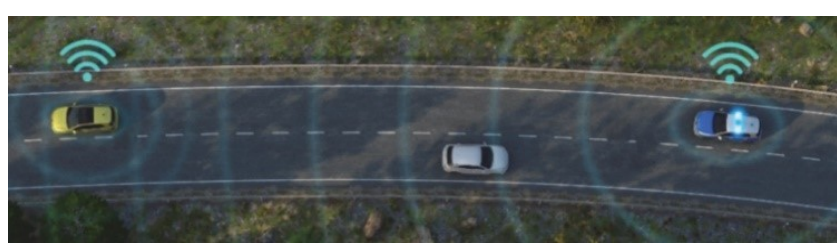

Fig 1. Wi-Fi solution in car-2-car communication

The WiFi solution has some drawbacks which must be overcome before this standard will be used on large scale. One of the most important is that each vehicle creates a virtual space around it where information can be exchanged (the WiFi range). There is a high volume of information which needs to be manipulated and filtered by each vehicle. This electromagnetic ,pollution” can highly increase in the crowded areas, and the possible interferences can decrease the communication performances.

The second main drawback is the security for safe data access, which is harder to be ensured in open wi-fi communication systems.

The current paper proposes an original solution which is based on the LiFi technology. This solution overcomes the drawbacks presented above and have several additional advantages: provides high transmission rates and it's performances are not decreasing in crowded areas [3].

The paper is structured as following: Section 2 presents the State-Of-the art in LiFi technology, Section 3 describes the proposed solution, Section 4 presents the experiments and Section 5 presents the Conclusion of the paper. 


\section{RELATED WORK}

The paper considers an original solution that can be used for car-2-car communication, based on LiFi technology. The solution can be integrated in the already available cars lightning systems. Using this type of system, the cars can communicate each-other by using their beams which are pulsing in such manner that can transmit information. The receiver for this transmission is an on-board camera which is able to detect beams from front-coming cars and read the sent information [3][11].

The feasibility study for this solution can be split in two parts:

- $\quad$ analyse the performances of the LiFi technology

- analyse the implementations of the existing technologies for cars beams management.

\section{A. LiFi on the market}

The LiFi (Light Fidelity) technology is based on the Visible Light Communications transmitting wireless internet data at very high speeds using only light beams. The LiFi technology was proposed initially by the German physicist Harald Haas during the Technology, Entertainment and Design Global Talk Conference (2011), after the invention of LED bulbs [5].

A LiFi network is very simple: it is needed only a light emitter on one end (i.e. an LED transmitter), and a photo sensitive detector (light sensor) on the other end. The data input to the LED transmitter is encoded into the light by varying the flickering rate (PWM) at which the LEDs turns "on" and "off" in order to generate different strings of bits of $1 \mathrm{~s}$ and $0 \mathrm{~s}$.

The LED intensity is modulated so rapidly that human eye cannot notice, so the light of the LED appears constant to humans. In fact, the current LED lights available for room lightning, or cars beams already use a PWM pulse to maintain the light intensity constant ignoring the voltage drops.

These types of systems are already available on the market offering gigabit speed for data transmission, but their applicability is currently limited to internet communication in closed environments. Even if the speed is above WiFi capabilities, they are sensitive to external noise (light) and therefore it is hard to be used in open environments. This can be considered an advantage in certain situations, as the network's security is more reliable and less liable on hacking.

There are several manufacturers on the market which offers complete solutions[6][8] and some universities have already adopted LiFi communications for their intranet [7].

\section{B. Automatic High Beam Management Systems}

The second question to which this paper tries to answer is if LiFi technology is feasible to be extended for car-2-car communication and what impact will have to the already existing cars architecture.

The current generation of high-end cars have solutions based on onboard cameras for automatically managing highbeams, called high-beams automatic assistants. The assistants recognize the oncoming vehicles during night, trace their direction and switches the headlights automatically from high beam to low beam when cars are approaching. This feature is very useful during night driving, making the activity much less stressful for the driver.

The intelligent headlight control is composed by an onboard camera for data acquisition and special image processing algorithms for measuring the ambient brightness and estimating the distance from vehicles placed in front and the oncoming traffic [9][10][11].

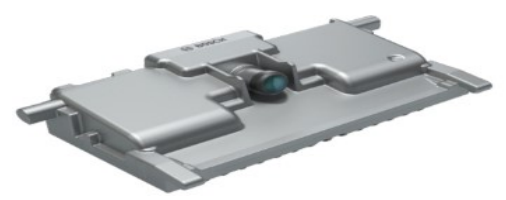

Fig 2. Sample multi-purpose camera used for High Beam assistance

The headlamps of the car are controlled by an electronic unit, based on the decisions taken after the captured images are processed. The road users who are in the beam range will be automatically excluded from the light distribution of the beam and put in a dark zone.

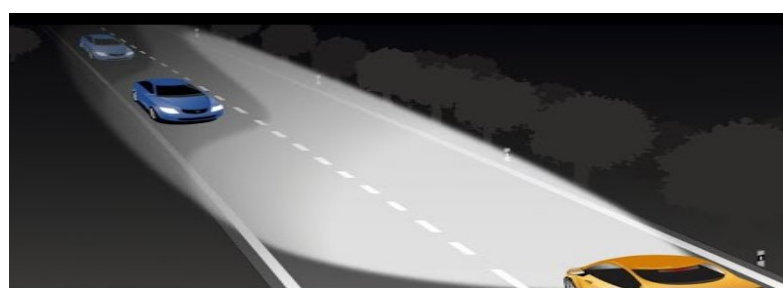

Fig 3. Light tunnel created to protect detected vehicles

Depending on the performances of the beamer, the high beam can be either turned off completely, or it can be dynamically configured to produce a "light tunnel" where the upcoming detected vehicle remains in the dark (e.g. LED matrix beamer)[10].

The solution considered in the paper is an adaption of the already existing technology, where the biggest change is in the software processing and the hardware changes are minimal (LED beams are already controlled via PWM, onboard camera for high-beams management is already available).

\section{SOLUTION OVERVIEW}

The feasibility of using a camera for car-2-car communication is presented in [11]. In the paper it is analysed the camera minimum performances needed to record communication from up-front coming cars. The conclusion of the paper was that a minimum frequency of $100 \mathrm{fps}$ is needed.

If a lower frequency would be used, the camera will not be able to record the communication in due time and with an acceptable error rate.

Although the current technology allows a top framerate of more than $1000 \mathrm{fps}$ for a camera, the capability is limited by the images processing speed needed to detect beams and read data.

\section{A. Beams Detection}

The solution implemented in this paper is based on the Open $\mathrm{CV}$. This is an open source computer vision and machine 
learning software library. It has more than 2500 basic and state-of-the-art computer vision and machine learning algorithms. It is cross platform with interfaces for $\mathrm{C}++$, Java, Python and MATLAB, runs on Windows, Linux, Android and Mac OS. OpenCV is mostly used for real-time vision applications.

The image processing algorithm from Open CV library was extended with a Python wrapper that allows detection of beams lights.

For detecting if the headlights are on or off, a simple approach is used. The continuous video data acquisition is split in sequential image frames which are independently processed, basically a continuous frame grabbing and processing. The algorithm performed for each image consists on several steps performed in loop: each image is transformed in a grayscale image; a thresholding function is applied afterwards to obtain a binary image. On this modified image is applied an edge detection or an area measurement. Based on the surface generated by the $\mathrm{ON}$ headlights the software can generate a distance using a scaling function applied on the continuous measurement of the image stream.

Once the beams are detected, further algorithms will be applied for monitoring and reading the data received from the beam light similar as in [3].

The advantage of this algorithm is that the processing time is reduced, and it can be applied during real-time acquisition process.

All the above functions, except the scaling of the image are by default implemented in Open CV. The needed adaption refers only to the threshold parameters which needs to be calibrated, depending on external environment conditions (e.g. fog light, day, night, snow, rain etc.)

Below, an example of the algorithm use can be observed.

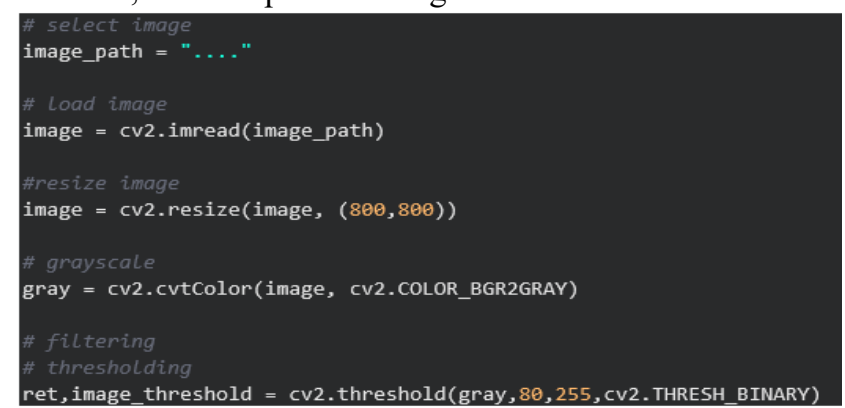

Fig 4. Python wrapper used for Image processing

When no beams are detected in the image, then the resulted image is completely black, those no size for the region.

\section{B. Experiments}

The scope of the experiments performed was to determine the accuracy of the algorithm for detecting beam lights in images, and to measure the processing time needed for this detection. This will directly influence the maximum frequency of the acquisition camera accepted by the system.

During testing it was used an images database which present cars having the beams on in different environment conditions: day, night, fog.
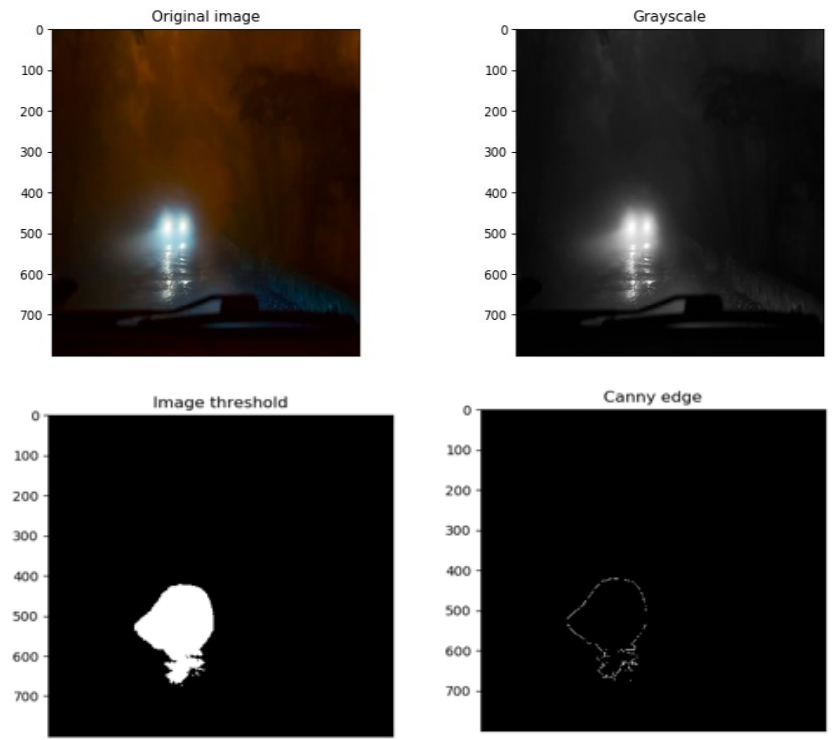

Fig 5. Image processing example

For the sets of images considered, the algorithm successfully detected the beams in images with a precision of more than $90 \%$ during night, and approx. 50\% during daytime.

Fig 6 presents the results obtained during region processing for the beams detection in different environments.

It was observed that the algorithm used obtains the best results during night, with low traffic conditions.

In order to increase the performances of the system, a multi-layer application should be considered, based on machine learning algorithms.

- the first layer only detects the general weather condition and, based on this, it is chosen which algorithm to be executed next for image processing

- the second layer performs beams detection using the algorithm decided during the execution of step 1 .

The second step of the experiment is to measure the processing time needed for one snapshot to detect the beams.

A full image processing operation is measured to be executed in average in $30 \mathrm{~ms}$ on a PC, considering a 800x800 pixels image.

Depending on the image resolution and environment data, the image processing times varies from $20 \mathrm{~ms}$ for a resolution of 600x600 up to more than $60 \mathrm{~ms}$ for a high traffic image.

No noticeable influence was observed by varying the image resolution.

Table 1 presents the average processing time measured for different images taken in different weather conditions and with different resolutions.

TABLE 1: Processing time measured

\begin{tabular}{|l|l|l|l|l|l|}
\hline Time (ms) & 20 & 29 & 62 & 32 & 45 \\
\hline Resolution & $600 \times 600$ & $800 \times 800$ & $800 \times 800$ & $800 \times 800$ & $1200 \times 1200$ \\
\hline Environment & $\begin{array}{l}\text { Night, } \\
\text { multiple } \\
\text { cars }\end{array}$ & $\begin{array}{l}\text { night, } \\
\text { single } \\
\text { car }\end{array}$ & $\begin{array}{l}\text { Night } \\
\text { multiple } \\
\text { cars }\end{array}$ & $\begin{array}{l}\text { Fog, } \\
\text { single } \\
\text { car }\end{array}$ & $\begin{array}{l}\text { night, } \\
\text { single car }\end{array}$ \\
\hline
\end{tabular}



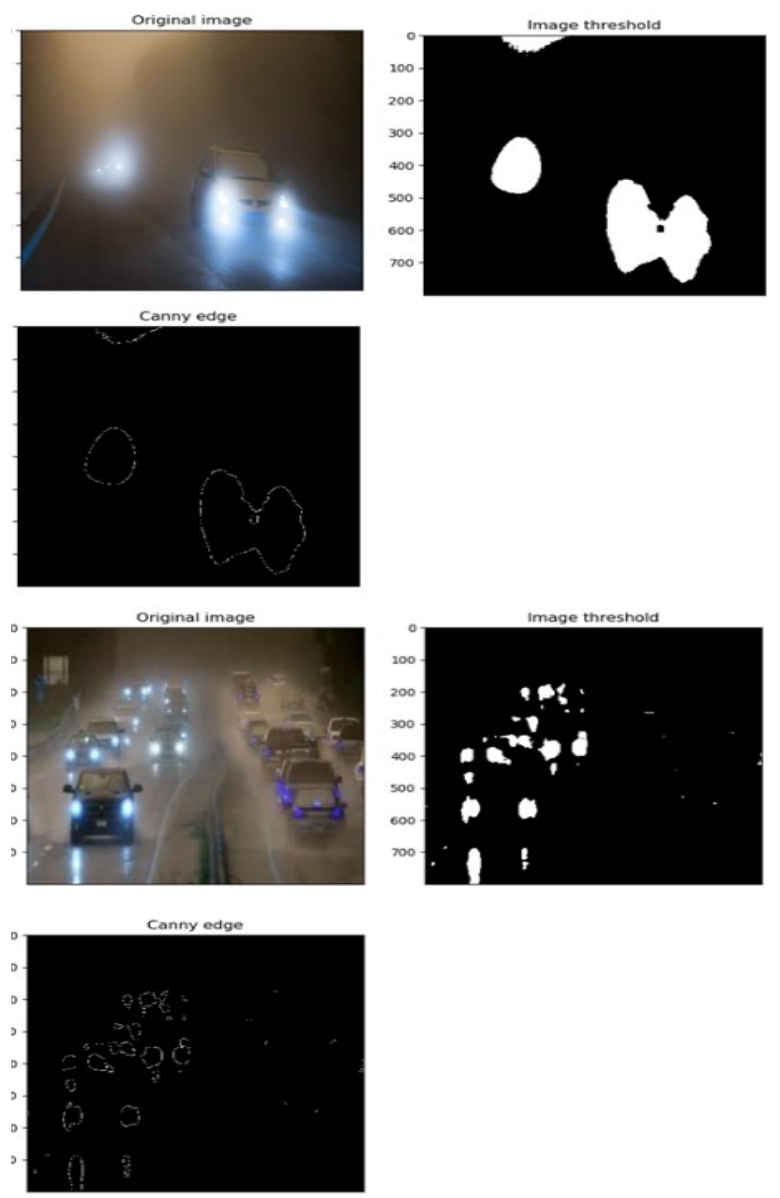

Fig 6. Image processing example

\section{System Improvements}

As it can be observed in the previous chapter, the average processing speed for the implemented algorithm is $40 \mathrm{~ms}$ for a 800x800 image resolution. That means the beams detection capability of the system will be of only 25 snapshots / sec.

Considering the results obtained in [11] it is needed a minimum framerate of $100 \mathrm{fps}$ in order that this technology to be feasible to be applied in real world.

Although at the first sight the two results are contradicting each other, this is not totally true. The detection algorithm is executed in loop only until on-coming beams are detected. Once they are detected, a second algorithm is started to trace beams direction, to read the flickering of beams and transform this into data. That means there is no need that the detection algorithm to be executed on a similar speed as camera acquisition speed.

The algorithm that needs to be implemented has 4 steps:

1. decide the beams detection algorithm that will be executed based on weather conditions

2. execute the beams detection algorithm decided on step 1 in loop until on-coming beams are detected in the camera visible range. The scope is to detect the region of the image where the beams are located
3. once the region is detected, the third step of the algorithm is started to trace the beams direction while there are in the visible range of the camera

4. the last step of the algorithm will read the flickering of the up-front vehicle beams and convert this into data

\section{CONCLUSION}

The paper presented an original solution that can be implemented for car-2-car communication based on $\mathrm{LiFi}$ technology and analyse the feasibility of such a system from images processing point of view.

The system is using the new generation of car illumination systems based on LED technology to encode and send the information to the other cars, and an onboard camera capable to detect beams from front-coming cars and record the sent data.

The on/off activity of the LED transmitter is totally invisible to human eye which do not perceive any flickering if a minimum frequency is used.

The demo application presented in this paper is based on the Open CV library for image processing and obtained good results in term of reliability and processing time.

However, in order to be adapted for an embedded system it will be needed an application based on $\mathrm{C}++$ interface of Open CV with different improvements for the runtime and application-oriented optimization.

\section{REFERENCES}

[1] Car 2 Car Communication Consortium https://www.car-2car.org/index.php?id=5

[2] https://www.car-2-car.org/fileadmin/press/pdf/volkswagen-localhazard-warning.pdf

[3] C. Stoica Spahiu, L. Stanescu and M. Brezovan, "Improving driver warnings accuracy using low-cost sensors," 2018 19th International Carpathian Control Conference (ICCC), Szilvasvarad, 2018, pp. 377 382, doi: 10.1109/CarpathianCC.2018.8399659.

[4] S. Eichler, C. Schroth, J. Eberspäche, "Car-to-Car Communication" Institute of Communication Networks, Technische Universität Vehicleto-Vehicle Communications:Readiness of V2V Technology for Application.US Department of transportation,National Traffic Safety administration 2014, DOT HS 812014

[5] H. Haas, "Opportunities and Challenges of Future LiFi," 2019 IEEE Photonics Conference (IPC), San Antonio, TX, USA, 2019, pp. 1-2.

[6] LiFi (Light Fidelity) \& its Applications, FN Division, TEC (2014) pureLiFi. [Online]. http://purelifi.com/

[7] LiFI applicability: https://purelifi.com/case-study/lifi-in-a-classroom/

[8] Oldedcomm LIFI systems:

https://www.oledcomm.net/category/products/lifinet-modemsaccessories/

[9] M. Alsumady, S. Alboon, "Intelligent Automatic High Beam Light Controller". Old City Publishing, Inc. Published by license under the OCP Science imprint, a member of the Old City Publishing Group., pp $1-8,2013$

[10] High Beam assistance:

https://www.hella.com/techworld/au/Technical/Automotivelighting/High-beam-assist-583/

[11] C.Stoica Spahiu, L.Stanescu, M.Brezovan, F.Petcusin, "LiFi Technology Feasibility Study for Car-2-Car Communication", 21st International Carpathian Control Conference, 2020 